\title{
DIE PHYSIKALISCHEN UND TECHNISCHEN GRUNDLAGEN DER HOCHFREQUENZBEHANDLUNG
}

\author{
DIATHERMIE · ARSONVALISATION · KURZWELLEN \\ VON \\ DR. MED. ET PHIL. HANS WEISZ
}

MIT EINEM GELEITWORT VON

J. KOWARSCHIK

MIT 36 TEXTABBILDUNGEN

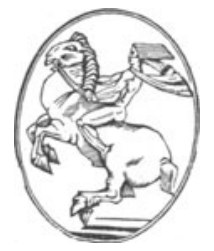

WIEN

VERLAG VON JULIUS SPRINGER 1935 
ISBN-13: 978-3-7091-9642-7 e-ISBN-13: 978-3-7091-9889-6

DOI: $10.1007 / 978-3-7091-9889-6$

ALLE RECHTE, INSBESONDERE DAS DER ÜBERSETZUNG IN FREMDE SPRACHEN, VORBEHALTEN

COPYRIGHT 1935 BY JULIUS SPRINGER IN VIENNA 


\section{Geleitwort.}

Unter den physikalischen Heilmethoden nimmt heute die Elektrotherapie den ersten Rang ein. Insbesondere ist es die Behandlung mit Hochfrequenzströmen, der in den letzten Jahren ein besonderes Interesse entgegengebracht wird. Gegenüber der Hochfrequenztherapie sind die alten Methoden der Elektrotherapie, wie Galvanisation und Faradisation, ganz in den Hintergrund getreten.

Die Entwicklung der Hochfrequenztherapie ist auf das engste mit den Fortschritten der Radiotechnik bzw. mit denen des Rundfunks verknüpft. Die Erfindung der Löschfunkenstrecke durch M. WIEN brachte uns den Übergang von der Arsonvalisation, der ältesten Methode der Hochfrequenzbehandlung, zur Diathermie. Während die Erfolge der Arsonvalisation noch vielfach umstritten waren, hat die Diathermie der Hochfrequenztherapie die allgemeine Anerkennung verschafft. Ihre biologische Wirksamkeit und ihre therapeutischen Erfolge konnten nicht mehr angezweifelt werden. Die Diathermie ist heute die verbreitetste Methode der Elektrotherapie. In den letzten Jahren hat sich nun in der Radiotechnik ein weiterer Fortschritt vollzogen, indem die Löschfunkenstrecke durch die Elektronenröhre ersetzt wurde. Dadurch ist es möglich geworden, Wechselströme sehr hoher Frequenz oder, was dasselbe ist, sehr kurzer Wellenlänge bei genügend hoher Leistung zu erzeugen. Dieser technische Fortschritt brachte uns in der Medizin die Kurzwellentherapie.

Aus diesen wenigen Tatsachen ist die enge Verbundenheit ersichtlich, die zwischen Hochfrequenztherapie und Hochfrequenztechnik besteht. Der Konstruktion eines neuen verbesserten Hochfrequenzgenerators folgt jeweils ein neuer Fortschritt in der Therapie. Arsonvalisation, Diathermie und Kurzwellentherapie kennzeichnen so drei technische Entwicklungsstufen.

Jeder, der das Wesen der Hochfrequenztherapie, ihre Apparate und deren Anwendung beherrschen will, muß sich daher mit den 
physikalischen und technischen Grundlagen dieser Methode vertraut machen. Im besonderen wird der wissenschaftlich arbeitende Arzt das Bedürfnis haben, die physikalisch-technischen Grundlagen etwas näher kennenzulernen. In den von Medizinern geschriebenen Lehrbüchern der Elektrotherapie, Diathermie und Kurzwellentherapie sind diese Grundlagen in der allerknappsten Form dargestellt. Sie können daher nur das bringen, was für den allgemeinen Praktiker unentbehrlich ist. Die von Physikern und Technikern verfaßten Werke lassen wohl an Ausführlichkeit nichts zu wünschen übrig, sind dafür aber in einer Sprache geschrieben, die dem Arzt so gut wie unverständlich ist. Die mathematisch-physikalischen Kenntnisse, welche diese Bücher voraussetzen, gehen weit über das durchschnittliche Wissen des Arztes hinaus. Die Gleichungen und Formeln, in welche sich ihre Lehren kleiden, vermögen ihm keine Vorstellungen zu vermitteln.

H. WeIsz hat es nun unternommen, diese physikalischmathematische Geheimsprache in ein auch dem Arzt verständliches Idiom zu übersetzen. Er schien für diese Aufgabe dadurch in besonderer Weise befähigt, daß er sowohl Arzt wie Physiker ist. Da er die Sprache beider kennt und beherrscht, ist er in der Lage, den Vermittler, den Dolmetsch zwischen ihnen abzugeben.

Die zum Teil nicht ganz einfachen Vorstellungen über das Wesen und die Wirkung der Hochfrequenzströme sind in dèm Büchlein auf die einfachste, jedem Laien verständliche Form gebracht, auf eine Form, die weder physikalische noch technische Kenntnisse zur Voraussetzung hat. Es wird so dem Arzt in leichter und müheloser Weise helfen, sein Wissen nach der physikalischen und technischen Seite hin zu erweitern. Für den Arzt wird ein solches Wissen nicht allein Selbstzweck sein, es wird in letzter Linie auch in einer Verbesserung seiner therapeutischen Technik und damit in einer Verbesserung seiner therapeutischen Erfolge zur Auswirkung kommen.

Wien, im Februar 1935.

J. Kowarschik. 


\section{Inhaltsverzeichnis.}

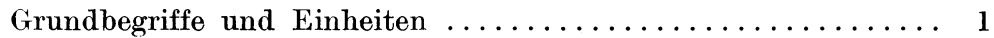

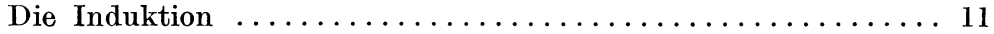

Der Wechselstrom ....................... 16

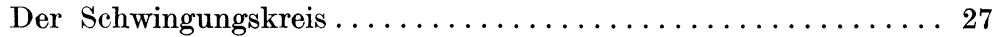

Ungedämpfte Schwingung und Elektronenröhre......... 33

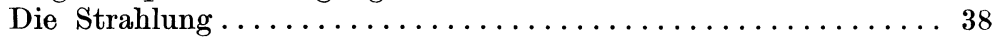

Durchgang hochfrequenter Ströme durch Leiter erster und

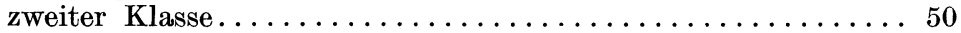

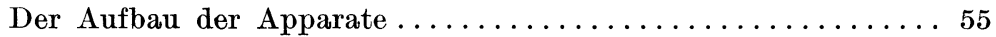

Behandlungstechnik ...................... 63 